\title{
Décisions médicales délicates
}

\section{Yvonne Gilli}

Dr méd., membre du Comité central de la FMH, responsable du département Numérisation/eHealth

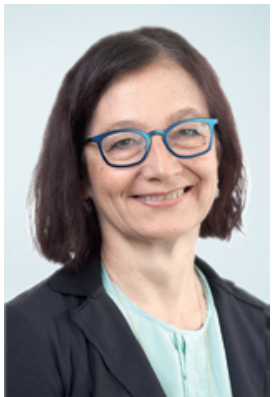

En médecine, certaines décisions peuvent s'avérer extrêmement délicates et placer les médecins face à un dilemme éthique. Spontanément, on pense aux décisions en fin de vie ou à des situations dans lesquelles le pronostic vital du patient est engagé, mais ces décisions peuvent aussi concerner des personnes particulièrement vulnérables qui, en raison de leur situation, ne peuvent pas disposer librement de la prise en charge à laquelle elles ont droit; et en particulier les requérants d'asile contraints de quitter notre pays suite à une décision de renvoi exécutoire.

Les tâches et responsabilités des médecins pénitentiaires lors de renvois sous contrainte ont été redéfinies dans le cadre d'un essai-pilote.

La dignité humaine est intangible. En tant qu'Etat de droit, la Suisse respecte les droits fondamentaux qui en découlent, dont le droit à une prise en charge médicale en présence d'éléments pouvant indiquer une menace pour la santé, ou si la personne concernée fait valoir une telle menace. En règle générale, ce sont les médecins pénitentiaires qui sont chargés d'évaluer l'état de santé sur la base de leurs connaissances médicales mais aussi des directives déontologiques et éthiques.

En 2013, l'ancien président de la Commission centrale d'éthique de l'Académie suisse des sciences médicales avait déjà soulevé la pratique problématique de l'accompagnement médical des rapatriements sous contrainte et exigé des améliorations [1]. Il a notamment critiqué l'absence de définition claire des tâches et des responsabilités et souligné le fait que, dans la plupart des cantons, les médecins pénitentiaires dépendent de la direction de la justice et de la prison et qu'ils peuvent donc être pris dans un conflit de loyauté entre la médecine d'une part et la dépendance à leur employeur de l'autre, à savoir l'autorité qui a ordonné le renvoi.

Depuis, un essai-pilote a été mené avec l'implication de tous les milieux concernés dans le but d'apporter des améliorations. Les médecins pénitentiaires rédigent désormais un rapport structuré confidentiel dans lequel ils consignent les facteurs de risques décelés, et examinent l'éventuelle présence de contre-indications au transport aérien d'après des directives internationales. Ils n'évaluent pas directement l'aptitude au transport, celle-ci relevant de la compétence de l'organisation en charge du renvoi qui met à disposition son propre expert médical. L'échange des informations médicales entre le médecin pénitentiaire et le médecin chargé du transport est assuré avec le consentement écrit de la personne à rapatrier. L'accompagnement médical du rapatriement est ainsi garanti et toute mesure de contrainte médicale est proscrite.

L'accompagnement médical dans le cadre de décisions de renvoi et de mesures de contrainte administrative demeure difficile. Les médecins pénitentiaires restent les employés des autorités ayant rendu la décision de renvoi, et à ce titre, ils sont tiraillés entre une décision exécutoire et l'éthique médicale, qui place la santé du patient au centre. En outre, la santé du patient ne dépend pas seulement du rapport médical rédigé avant le renvoi, mais également de la prise en charge médicale future lors du retour dans le pays d'origine. Or nous savons tous que la plupart des personnes expulsées n'auront plus la garantie d'accéder à des soins une fois dans leur pays. Pour beaucoup, la seule acquisition de médicaments efficaces pourra s'avérer véritablement problématique, voire impossible.

\section{L'évaluation médicale de personnes contraintes de quitter notre pays reste une décision particulièrement délicate.}

En ce sens, l'évaluation médicale de personnes contraintes de quitter notre pays reste une décision particulièrement délicate. Autant les instances responsables que les médecins se doivent de réfléchir aux conditions-cadres. A titre individuel, le médecin est systématiquement face à un cas de conscience qui le place à la frontière entre responsabilité éthique et son rôle face aux décisions d'un Etat de droit. 